\title{
SHARP BOUNDS FOR SEIFFERT MEANS IN TERMS OF LEHMER MEANS
}

\section{MiaO-Kun Wang, Ye-FAng QIU And Yu-Ming ChU}

Abstract. In this paper, we establish two sharp inequalities as follows: $P(a, b)>L_{-\frac{1}{6}}(a, b)$ and $T(a, b)<L_{\frac{1}{3}}(a, b)$ for all $a, b>0$ with $a \neq b$. Here, $L_{r}(a, b), P(a, b)$ and $T(a, b)$ are the Lehmer, first and second Seiffert means of $a$ and $b$, respectively.

Mathematics subject classification (2010): 26E60.

Keywords and phrases: Lehmer mean, first Seiffert mean, second Seiffert mean.

\section{REFERENCES}

[1] D. H. LeHMER, On the compunding of certain means, J. Math. Anal. Appl., 36 (1971), 183-200.

[2] H. ALZER, Über Lehmers Mittelwertfamilie, Elem. Math., 43, 2 (1988), 50-54.

[3] ZH. LIU, Remark on inequalities between Hölder and Lehmer means, J. Math. Anal. Appl., 247, 1 (2000), 309-313.

[4] H. AlzER, Bestmögliche Abschätzungen für spezielle Mittelwerte, Zb. Rad. Prirod. -Mat. Fak. Ser. Mat., 23, 1 (1993), 331-346.

[5] K. B. Stolarsky, Hölder means, Lehmer means, and $x^{-1} \log \cosh x$, J. Math. Anal. Appl., 202, 3 (1996), 810-818.

[6] H. J. SEIFFERT, Problem 887, Nieuw Arch. Wisk. (4), 11 (1993), 176-176.

[7] H. J. SEIFFERT, Aufgabe $\beta 16$, Die Wurzel, 29 (1995), 221-222.

[8] P. A. Ḧ̈sтӧ, Optimal inequalities between Seiffert's mean and power mean, Math. Inequal. Appl., 7, 1 (2004), 47-53.

[9] E. NEUMAn, J. SÁNDOR, On certain means of two arguments and their extensions, Int. J. Math. Math. Sci., 16 (2003), 981-983.

[10] E. Neuman, J. SÁndor, On the Schwab-Borchardt mean, Math. Pannon., 14, 2 (2003), $253-266$.

[11] P. A. Ḧ̈s T̈̈, A monotonicity property of ratios of symmetric homogeneous means, JIPAM. J. Inequal. Pure Appl. Math., 3, 5 (2002), Article 71, 23 pp.

[12] P. S. Bullen, D. S. Mitrinović And P. M. Vasić, Means and their inequalities, D. Reidel Publishing Co., Dordrecht, 1988. 